\title{
Traveling Solitary Wave Solutions for the Symmetric Regularized Long-Wave Equation
}

Mahmoud A.E. Abdelrahman and Mostafa M.A. Khater

Mansoura University,Department of Mathematics, Faculty of Science, 35516 Mansoura, Egypt.

\section{ABSTRACT}

mostafa.khater2024@yahoo.com

\begin{abstract}
In this paper, we employ the extended tanh function method to find the exact traveling wave solutions involving parameters of the symmetric regularized long- wave equation. When these parameters are taken to be special values, the solitary wave solutions are derived from the exact traveling wave solutions. These studies reveal that the symmetric regularized long-wave equation has a rich variety of solutions.
\end{abstract}

\section{Keywords}

The extended tanh function method; The symmetric regularized long-wave equation; Traveling wave solutions; Solitary wave solutions.

\section{Council for Innovative Research}

Peer Review Research Publishing System

Journal: JOURNAL OF ADVANCES IN MATHEMATICS

Vol .11, No.8

www.cirjam.com, editorjam@gmail.com 


\section{INTRODUCTION}

The nonlinear partial differential equations of mathematical physics are major subjects in physical science [1]. Exact solutions for these equations play an important role in many phenomena in physics such as fluid mechanics, hydrodynamics, Optics, Plasma physics and so on. Recently many new approaches for finding these solutions have been proposed, for example, tanh - sech method [2]-[4], extended tanh - method [5]-[7], sine - cosine method [8]-[10], modified simple equation method $[11,12]$, the $\exp (\exp (-\phi(\xi)))$-expansion method [13]-[15], the extended $\exp (\exp (-\phi(\xi)))$ - expansion method [16], $\left(\frac{G}{G}\right)$-expansion method [17]-[19], Jacobi elliptic function method [20]- [23] and so on.

The objective of this article is to apply The extended tanh function method for finding the exact traveling wave solution of the symmetric regularized long- wave equation Which describe shallow water waves and plasma drift waves. The rest of this paper is organized as follows: In Section 2, we give the description of the extended Jacobi elliptic function expansion method In Section 3, we use this method to find the exact solutions of the nonlinear evolution equations pointed out above. In Section 4, conclusions are given.

\section{Description of method}

Consider the following nonlinear evolution equation

$$
P\left(u_{,} u_{t}, u_{x}, u_{t t}, u_{x x^{\prime}} \ldots . .\right)=0_{x}
$$

Since, $\mathrm{P}$ is a polynomial in $u(x, t)$ and its partial derivatives. In the following, we give the main steps of this method.

Step 1. We use the traveling wave solution in the form

$$
u(x, t)=u(\xi), \quad \xi=x-c t,
$$

Where $\mathrm{c}$ is a positive constant, to reduce Eq. (2.1) to the following ODE:

$$
p\left(u, u^{t}, u^{n}, u^{n s}, \ldots . . .\right)=0 \text {, }
$$

Where $\mathrm{P}$ is a polynomial in $\mathrm{u}(\xi)$ and its total derivatives, while $u^{\prime}=\frac{d u}{d \xi}$.

Step 2. Suppose that the solution of ODE (2.3) can be expressed

$$
u(\xi)=a_{0}+\sum_{i=0}^{M}\left(a_{i} \varphi^{i}+b_{i} \varphi^{-1}\right)
$$

where $a_{i}, b_{i}$ are arbitrary constants to be determined, such that $a_{m} \neq 0$ or $b_{m} \neq 0$, while $\varphi$ satisfies the Riccati equation

$$
\varphi^{\prime}=b+\varphi^{2}
$$

where $b$ is a constant. Eq.(2.5) admits several types of solutions according to

Case 1. If $b<0$, then

$$
\varphi=-\sqrt{-b} \tanh (\sqrt{-b} \xi), \text { or } \varphi=-\sqrt{-b} \operatorname{coth}(\sqrt{-b} \xi) .
$$

Case 2. If $b>0$, then

$$
\varphi=\sqrt{b} \tan (\sqrt{b} \xi), \operatorname{or} \varphi=\sqrt{b} \cot (\sqrt{b} \xi)
$$


Case 3. If $b=0$, then

$$
\varphi=-\frac{1}{\xi}
$$

Step 3. Determine the positive integer $m$ in Eq.(1.4) by balancing the highest order derivatives and the nonlinear terms.

Step 4. Substitute Eq.(2.4) along Eq.(2.5) into Eq.(2.3) and collecting all the terms of the same power $\left(\varphi^{i}, i=0, \pm 1, \pm 2, \pm 3, \ldots\right)$ and equating them to zero, we obtain a system of algebraic equations, which can be solved by Maple or Mathematica to get the values of $a_{i}$ and $b_{i}$.

Step 5. Substituting these values and the solutions of Eq.(2.5) into Eq.(2.4) we obtain the exact solutions of Eq.(2.1).

\section{The SRLW equation}

Here, we will apply the extended tanh function method described in Sec.2 to find the exact traveling wave solutions and then the solitary wave solutions The SRLW equation [24].

Consider the SRLW equation is in the form

$v_{t t}-v_{x x}+\left(\frac{v^{2}}{2}\right)_{x t}-v_{x x t t}=0$,

by using the transformation $v(\xi)=v(x, t)$ since $\xi=x+k t$. Where $\mathrm{k}$ is arbitrary constant to be determined later, we get

$\left(k^{2}-1\right) v^{s}-k\left(\frac{v^{2}}{2}\right)^{s}-k^{2} v^{n ! v}=0$.

By integration Eq.(3.2) twice with negligence of integral constant, we get $\left(k^{2}-1\right) v-\frac{k}{2} v^{2}-k^{2} v^{v}=0$.

Balancing $v^{\prime \prime}$ and $v^{2} \Rightarrow m=2$, so that, we assume the solution of Eq.(3.3) be in the form

$$
v(\xi)=a_{0}+a_{1} \phi+a_{2} \phi^{2}+\frac{b_{1}}{\phi}+\frac{b_{2}}{\phi^{2}} .
$$

Substituting Eq.(3.4) and it's derivatives into Eq.(3.3) and collecting the coefficients of $\phi^{i}{ }_{s} i=0, \pm 1, \pm 2, \ldots$ and set it to zero we obtain the system of equation

$$
\left\{\begin{array}{c}
\frac{-1}{2} k a_{2}^{2}-6 k^{2} a_{2}=0 \\
-k a_{1} a_{2}-2 k^{2} a_{1}=0 \\
\left(k^{2}-1\right) a_{2}-\frac{1}{2} k\left(a_{1}^{2}+2 a_{0} a_{2}\right)-8 k^{2} a_{2} b=0 \\
\left(k^{2}-1\right) a_{1}-\frac{1}{2} k\left(2 a_{0} a_{1}+2 a_{2} b_{1}\right)-2 k^{2} a_{1} b=0 \\
\left(k^{2}-1\right) a_{0}-\frac{1}{2} k\left(a_{0}^{2}+2 a_{2} b_{2}+2 a_{1} b_{1}\right)-k^{2}\left(2 b_{2}+2 a_{2} b^{2}\right)=0 \\
\left(k^{2}-1\right) b_{1}-\frac{1}{2} k\left(2 a_{0} b_{1}+2 a_{1} b_{2}\right)-2 k^{2} b_{1} b=0 \\
\left(k^{2}-1\right) b_{2}-\frac{1}{2} k\left(b_{1}^{2}+2 a_{0} b_{2}\right)-8 k^{2} b_{2} b=0 \\
-k b_{1} b_{2}-2 k^{2} b_{1} b^{2}=0 \\
-\frac{1}{2} k b_{2}^{2}-6 k^{2} b_{2} b^{2}=0
\end{array}\right.
$$

Solving above system by using Maple 16, we get 


\section{Case 1.}

$b=-\frac{1}{4} \frac{k^{2}-1}{k^{2}}, a_{0}=3 \frac{k^{2}-1}{k}, a_{1}=0, a_{2}=-12 k, b_{1}=0, b_{2}=0$.

\section{Case 2.}

$b=\frac{1}{4} \frac{k^{2}-1}{k^{2}}, a_{0}=\frac{1-k^{2}}{k}, a_{1}=0, a_{2}=-12 k, b_{1}=0, b_{2}=0$.

\section{Case 3.}

$b=-\frac{1}{16} \frac{k^{2}-1}{k^{2}}, a_{0}=\frac{3}{2} \frac{k^{2}-1}{k}, a_{1}=0, a_{2}=-12 k, b_{1}=0, b_{2}=-\frac{3}{64} \frac{k^{4}-2 k^{2}+1}{k^{3}}$.

\section{Case 4.}

$b=\frac{1}{16} \frac{k^{2}-1}{k^{2}}, a_{0}=\frac{1}{2} \frac{k^{2}-1}{k}, a_{1}=0, a_{2}=-12 k, b_{1}=0, b_{2}=-\frac{3}{64} \frac{k^{4}-2 k^{2}+1}{k^{3}}$.

\section{Case 5.}

$b=-\frac{1}{4} \frac{k^{2}-1}{k^{2}}, a_{0}=3 \frac{k^{2}-1}{k}, a_{1}=0, a_{2}=0, b_{1}=0, b_{2}=-\frac{3\left(k^{2}-1\right)^{2}}{4}$.

\section{Case 6.}

$b=\frac{1}{4} \frac{k^{2}-1}{k^{2}}, a_{0}=-\frac{k^{2}-1}{k}, a_{1}=0, a_{2}=0, b_{1}=0, b_{2}=\frac{-3}{4} \frac{\left(k^{2}-1\right)^{2}}{k^{3}}$.

So that, we will study each case and get the exact traveling wave solution and also the solitary wave solutions for Eq. (3.3).

\section{For Case 1.}

The exact traveling wave solution is in the form:

$v(\xi)=3 \frac{k^{2}-1}{k}-12 k \phi^{2}$.

The solitary wave solution is in the form:

Case i. If $b<0$, we get

$v(\xi)=3 \frac{k^{2}-1}{k}-12 k(-\sqrt{-b} \tanh (\sqrt{-b} \xi))^{2}$.

Or

$v(\xi)=3 \frac{k^{2}-1}{k}-12 k(-\sqrt{-b} \operatorname{coth}(\sqrt{-b} \xi))^{2}$.

Case ii. If $b>0$, we get

$v(\xi)=3 \frac{k^{2}-1}{k}-12 k(\sqrt{b} \tan (\sqrt{b} \xi))^{2}$.

Or

$v(\xi)=3 \frac{k^{2}-1}{k}-12 k(\sqrt{b} \cot (\sqrt{b} \xi))^{2}$.

Case iii. If $b=0$, we get 
$v(\xi)=3 \frac{k^{2}-1}{k}-12 k\left(\frac{1}{\xi}\right)^{2}$.

\section{For Case 2.}

The exact traveling wave solution is in the form:

$v(\xi)=\frac{k^{2}-1}{k}-12 k \phi^{2}$.

The solitary wave solution is in the form:

Case i. If $b<0$, we get

$v(\xi)=\frac{k^{2}-1}{k}-12 k(-\sqrt{-b} \tanh (\sqrt{-b} \xi))^{2}$.

Or

$v(\xi)=\frac{k^{2}-1}{k}-12 k(-\sqrt{-b} \operatorname{coth}(\sqrt{-b} \xi))^{2}$.

Case ii. If $b>0$, we get

$v(\xi)=\frac{k^{2}-1}{k}-12 k(\sqrt{b} \tan (\sqrt{b} \xi))^{2}$.

Or

$v(\xi)=\frac{k^{2}-1}{k}-12 k(\sqrt{b} \cot (\sqrt{b} \xi))^{2}$.

Case iii. If $b=0$, we get

$v(\xi)=\frac{k^{2}-1}{k}-12 k\left(\frac{1}{\xi}\right)^{2}$.

\section{For Case 3.}

The exact traveling wave solution is in the form:

$v(\xi)=\frac{3}{2} \frac{k^{2}-1}{k}-12 k \phi^{2}-\frac{3}{64} \frac{k^{4}-2 k^{2}+1}{k^{3}} \frac{1}{\phi^{2}}$.

The solitary wave solution is in the form:

Case i. If $b<0$, we get

$v(\xi)=\frac{3}{2} \frac{k^{2}-1}{k}-12 k(-\sqrt{-b} \tanh (\sqrt{-b} \xi))^{2}-\frac{3}{64} \frac{k^{4}-2 k^{2}+1}{k^{3}} \frac{1}{(-\sqrt{-b} \tanh (\sqrt{-b} \xi))^{2}}$.

Or

$v(\xi)=\frac{3}{2} \frac{k^{2}-1}{k}-12 k(-\sqrt{-b} \operatorname{coth}(\sqrt{-b} \xi))^{2}-\frac{3}{64} \frac{k^{4}-2 k^{2}+1}{k^{3}} \frac{1}{(-\sqrt{-b} \operatorname{coth}(\sqrt{-b} \xi))^{2}}$

Case ii. If $b>0$, we get 
$v(\xi)=\frac{3}{2} \frac{k^{2}-1}{k}-12 k(\sqrt{b} \tan (\sqrt{b} \xi))^{2}-\frac{3}{64} \frac{k^{4}-2 k^{2}+1}{k^{3}} \frac{1}{(\sqrt{b} \tan (\sqrt{b} \xi))^{2}}$

Or

$v(\xi)=\frac{3}{2} \frac{k^{2}-1}{k}-12 k(\sqrt{b} \cot (\sqrt{b} \xi))^{2}-\frac{3}{64} \frac{k^{4}-2 k^{2}+1}{k^{3}} \frac{1}{(\sqrt{b} \cot (\sqrt{b} \xi))^{2}}$

Case iii. If $b=0$, we get

$v(\xi)=\frac{3 k^{2}-1}{2}-12 k\left(\frac{1}{\xi}\right)^{2}-\frac{3}{64} \frac{k^{4}-2 k^{2}+1}{k^{3}} \frac{1}{\left(\frac{1}{\xi}\right)^{2}}$

\section{For Case 4.}

The exact traveling wave solution is in the form:

$v(\xi)=\frac{1}{2} \frac{k^{2}-1}{k}-12 k \phi^{2}-\frac{3}{64} \frac{k^{4}-2 k^{2}+1}{k^{3}} \frac{1}{\phi^{2}}$.

The solitary wave solution is in the form:

Case i. If $b<0$, we get

$v(\xi)=\frac{1}{2} \frac{k^{2}-1}{k}-12 k(-\sqrt{-b} \tanh (\sqrt{-b} \xi))^{2}-\frac{3}{64} \frac{k^{4}-2 k^{2}+1}{k^{3}} \frac{1}{(-\sqrt{-b} \tanh (\sqrt{-b} \xi))^{2}}$.

Or

$v(\xi)=\frac{1}{2} \frac{k^{2}-1}{k}-12 k(-\sqrt{-b} \operatorname{coth}(\sqrt{-b} \xi))^{2}-\frac{3}{64} \frac{k^{4}-2 k^{2}+1}{k^{3}} \frac{1}{(-\sqrt{-b} \operatorname{coth}(\sqrt{-b} \xi))^{2}}$

Case ii. If $b>0$, we get

$v(\xi)=\frac{1}{2} \frac{k^{2}-1}{k}-12 k(\sqrt{b} \tan (\sqrt{b} \xi))^{2}-\frac{3}{64} \frac{k^{4}-2 k^{2}+1}{k^{3}} \frac{1}{(\sqrt{b} \tan (\sqrt{b} \xi))^{2}}$

Or

$v(\xi)=\frac{1}{2} \frac{k^{2}-1}{k}-12 k(\sqrt{b} \cot (\sqrt{b} \xi))^{2}-\frac{3}{64} \frac{k^{4}-2 k^{2}+1}{k^{3}} \frac{1}{(\sqrt{b} \cot (\sqrt{b} \xi))^{2}}$

Case iii. If $b=0$, we get

$v(\xi)=\frac{1}{2} \frac{k^{2}-1}{k}-12 k\left(\frac{1}{\xi}\right)^{2}-\frac{3}{64} \frac{k^{4}-2 k^{2}+1}{k^{3}} \frac{1}{\left(\frac{1}{\xi}\right)^{2}}$.

\section{For Case 5.}

The exact traveling wave solution is in the form: 
$v(\xi)=3 \frac{k^{2}-1}{k}-\frac{3}{4} \frac{\left(k^{2}-1\right)^{2}}{k^{3}} \frac{1}{\phi^{2}}$.

The solitary wave solution is in the form:

Case i. If $b<0$, we get

$v(\xi)=3 \frac{k^{2}-1}{k}-\frac{3}{4} \frac{\left(k^{2}-1\right)^{2}}{k^{3}} \frac{1}{(-\sqrt{-b} \tanh (\sqrt{-b} \xi))^{2}}$

Or

$v(\xi)=3 \frac{k^{2}-1}{k}-\frac{3}{4} \frac{\left(k^{2}-1\right)^{2}}{k^{3}} \frac{1}{(-\sqrt{-b} \operatorname{coth}(\sqrt{-b} \xi))^{2}}$.

Case ii. If $b>0$, we get

$v(\xi)=3 \frac{k^{2}-1}{k}-\frac{3}{4} \frac{\left(k^{2}-1\right)^{2}}{k^{3}} \frac{1}{(\sqrt{b} \tan (\sqrt{b} \xi))^{2}}$

Or

$v(\xi)=3 \frac{k^{2}-1}{k}-\frac{3}{4} \frac{\left(k^{2}-1\right)^{2}}{k^{3}} \frac{1}{(\sqrt{b} \cot (\sqrt{b} \xi))^{2}}$.

Case iii. If $b=0$, we get

$v(\xi)=3 \frac{k^{2}-1}{k}-\frac{3}{4} \frac{\left(k^{2}-1\right)^{2}}{k^{3}} \frac{1}{\left(\frac{1}{\xi}\right)^{2}}$.

\section{For Case 6.}

The exact traveling wave solution is in the form:

$v(\xi)=-\frac{k^{2}-1}{k}-\frac{3}{4} \frac{\left(k^{2}-1\right)^{2}}{k^{3}} \frac{1}{\phi^{2}}$.

The solitary wave solution is in the form:

Case i. If $b<0$, we get

$v(\xi)=-\frac{k^{2}-1}{k}-\frac{3}{4} \frac{\left(k^{2}-1\right)^{2}}{k^{3}} \frac{1}{(-\sqrt{-b} \tanh (\sqrt{-b} \xi))^{2}}$.

Or

$v(\xi)=-\frac{k^{2}-1}{k}-\frac{3}{4} \frac{\left(k^{2}-1\right)^{2}}{k^{3}} \frac{1}{(-\sqrt{-b} \operatorname{coth}(\sqrt{-b} \xi))^{2}}$.

Case ii. If $b>0$, we get 


$$
v(\xi)=-\frac{k^{2}-1}{k}-\frac{3}{4} \frac{\left(k^{2}-1\right)^{2}}{k^{3}} \frac{1}{(\sqrt{b} \tan (\sqrt{b} \xi))^{2}}
$$

Or

$v(\xi)=-\frac{k^{2}-1}{k}-\frac{3}{4} \frac{\left(k^{2}-1\right)^{2}}{k^{3}} \frac{1}{(\sqrt{b} \cot (\sqrt{b} \xi))^{2}}$

Case iii. If $b=0$, we get

$$
v(\xi)=-\frac{k^{2}-1}{k}-\frac{3}{4} \frac{\left(k^{2}-1\right)^{2}}{k^{3}} \frac{1}{\left(\frac{1}{\xi}\right)^{2}}
$$

\section{Remark:}

All the obtained results have been checked with Maple 16 by putting them back into the original equation and found correct.

\section{Acknowledgement}

The author thanks the referees for their suggestions and comments.

\section{Conclusion}

The extended tanh function method has been applied in this paper to find the exact traveling wave solutions and then the solitary wave solutions of the symmetric regularized long-wave equation. Let us compare between our results obtained in the present article with the well-known results obtained by other authors using different methods as follows: Our results of the symmetric regularized long-wave equation are new and different from those obtained in [28]. The obtained exact solutions can be used as benchmarks against the numerical simulations in theoretical physics and fluid mechanics.

\section{References}

[1] M. J. Ablowitz, H. Segur, Solitions and Inverse Scattering Transform, SIAM, Philadelphia 1981.

[2] W. Maliet, Solitary wave solutions of nonlinear wave equation, Am. J. Phys., 60 (1992) 650-654.

[3] W. Maliet, W. Hereman, The tanh method: Exact solutions of nonlinear evolution and wave equations, Phys.Scr., 54 (1996) 563-568.

[4] A. M. Wazwaz, The tanh method for traveling wave solutions of nonlinear equations, Appl. Math. Comput., 154 (2004) 714-723.

[5] S. A. EL-Wakil, M.A.Abdou, New exact traveling wave solutions using modified extended tanh-function method, Chaos Solitons Fractals, 31 (2007) 840-852.

[6] Mostafa M. A. Khater, Emad H. M. Zahran, New solitary wave solution of the generalized Hirota-Satsuma couple KdV system, International Journal of Scientific Engineering Research, Volume 6, Issue 8, August (2015)

[7] Mahmoud A.E. Abdelrahman, Emad H. M. Zahran and Mostafa M.A. Khater, Exact Traveling Wave Solutions for Modi_ed Liouville Equation Arising in Mathematical Physics and Biology. International Journal of Computer Applications (0975-8887) Volume 112 - No. 12, February (2015).

[8] A. M. Wazwaz, Exact solutions to the double sinh-Gordon equation by the tanh method and a variable separated ODE. method, Comput. Math. Appl., 50 (2005) 1685-1696.

[9] A. M.Wazwaz, A sine-cosine method for handling nonlinear wave equations, Math. Comput. Modelling, 40 (2004) 499 508.

[10] C. Yan, A simple transformation for nonlinear waves, Phys. Lett. A 224 (1996) 77-84.

[11] Mostafa M. A. Khater, the Modi_ed Simple Equation Method and its Applications in Mathematical Physics and Biology, Global Journal of Science Frontier Research: F Mathematics and Decision Sciences, Volume 15 Issue 4 Version $1.0(2015)$.

[12] Emad H. M. Zahran and Mostafa M.A. Khater, The modi_ed simple equation method and its applications for solving some nonlinear evolutions equations in mathematical physics. Jokull journal Vol. 64. Issue 5, 297-312. May (2014). 
[13] Mahmoud A.E. Abdelrahman, Emad H. M. Zahran and Mostafa M.A. Khater, Exact traveling wave solutions for power law and Kerr law non linearity using the $\exp (\exp (-\phi(\xi)))$ - expansion method. GJSFR Volume 14-F Issue 4 Version 1.0 (2014).

[14] Mahmoud A.E. Abdelrahman and Mostafa M.A. Khater, the $\operatorname{Exp}(\exp (-\phi(\xi)))-$ Expansion

Method and its Application for Solving Nonlinear Evolution Equations. International Journal of Science and Research (IJSR) ISSN (Online): 2319-7064, Volume 4 Issue 2, 2143-2146. February (2015).

[15] Mahmoud A.E. Abdelrahman, Emad H. M. Zahran and Mostafa M.A. Khater, the exp $(\exp (-\phi(\xi)))-$ Expansion Method and Its Application for Solving Nonlinear Evolution Equations. International Journal of Modern Nonlinear Theory and Application, 4, 37-47(2015).

[16] Mostafa M. A. Khater, Extended $\exp (\exp (-\phi(\xi)))$ - Expansion method for Solving the Generalized HirotaSatsuma Coupled KdV System,GJSFR-F Volume 15 Issue 7 Version 1.0 (2015).

[17] M. L.Wang, J. L. Zhang, X. Z. Li, The $\left(\frac{G}{G}\right)$-expansion method and traveling wave solutions of nonlinear evolutions equations in mathematical physics, Phys. Lett. A 372 (2008) 417-423.

[18] S. Zhang, J. L. Tong, W.Wang, A generalized $\left(\frac{G l}{G}\right)$ - expansion method for the mKdv equation with variable coe_cients, Phys. Lett. A 372 (2008) 2254-2257.

[19] Emad H.M. Zahran and Mostafa M. A. Khater, Exact solution to some nonlinear evolution equations by The $\left(\frac{G}{G}\right)-$ expansion method, Jokull journal Vol. 64, issue.5, (2014).

[20] S.I Zaki, Solitary wave interactions for the modified equal width wave equation, Comput. Phys. Commun. 126:219-213 (2000).

[21] Emad H. M. Zahran and Mostafa M.A. Khater, Exact Traveling Wave Solutions for the System of Shallow Water Wave Equations and Modified Liouville Equation Using Extended Jacobian Elliptic Function Expansion Method. American Journal of Computational Mathematics, 4, 455-463(2014).

[22] Emad H. M. Zahran Mostafa M. A. Khater, Extended Jacobian Elliptic Function Expansion Method and Its Applications in Biology. Applied Mathematics, 6, 1174- 1181 (2015).

[23] X. Q. Zhao, H.Y.Zhi, H.Q.Zhang, Improved Jacobi-function method with symbolic computation to construct new double-periodic solutions for the generalized Ito system, Chaos Solitons Fractals, 28 (2006) 112-126.

[24] Fakir Chand *, Anand K Malik, Exact Traveling Wave Solutions of Some Nonlinear Equations Using $\left(\frac{G}{G}\right)$-expansion Method methods, International Journal of Nonlinear Science

Vol.14(2012) No.4,pp.416-424 